\title{
(5) \\ Violencia y depresión en estudiantes universitarios en el contexto de la COVID-19
}

Violence and depression in university students in the context of COVID-19

Violência e depressão em estudantes universitários no contexto do COVID-19

\author{
Martha Quevedo \\ Universidad Autónoma de Guerrero, México \\ https://orcid.org/0000-0003-4997-6197 \\ Kenny Acuña \\ Universidad Autónoma de Guerrero, México \\ https://orcid.org/0000-0002-3686-7138
}

DOI: https://doi.org/10.35622/j.rep.2021.03.004

Recibido 02/12/2020/ Aceptado 22/07/2021

RESUMEN. El presente estudio tuvo como propósito conocer la relación existente entre la violencia de pareja y la depresión en estudiantes de la Universidad Autónoma de Guerrero, México, durante la pandemia COVID-19. La investigación es mixta, se aplicaron cuestionarios con respuestas de opción múltiple que derivaron del Inventario de Depresión de Beck y el Test de Violencia. La población estaba constituida por 737 estudiantes, 564 eran mujeres y 173 hombres. De estos, el $71.91 \%$ expresó contar con una saludable relación de pareja, el 8.82\% presentó violencia leve y el 6.92\% violencia grave, sin embargo, el $29.44 \%$ mostró ideación suicida. Los resultados indican que los universitarios mostraron casos de depresión y sólo la cuarta parte mostró pensamiento suicida. Esto quiere decir que la crisis sanitaria sí afectó el estado mental de los estudiantes y con esto un problema académico que debe ser resuelto por las autoridades universitarias.

PALABRAS CLAVE: Depresión, estudiantes, pandemia, violencia.

ABSTRACT. The purpose of the present study was to know the relationship between intimate partner violence and depression in students of the Autonomous University of Guerrero, Mexico, during the COVID-19 pandemic. The research is mixed; questionnaires with multiple-choice responses derived from the Beck Depression Inventory and the Violence Test were applied. The population consisted of 737 students; 564 were women, and 173 were men. Of these, $71.91 \%$ expressed having a healthy relationship with their partner, $8.82 \%$ presented mild violence, and $6.92 \%$ severe violence; however, $29.44 \%$ showed suicidal ideation. The results indicate that the university students showed cases of depression, and only a quarter showed suicidal thoughts. This means that the health crisis did affect the mental state of the students, and with this an academic problem that the university authorities must resolve.

KEYWORDS: Depression, students, pandemic, violence.

RESUM0. 0 objetivo do presente estudo foi conhecer a relação entre violência por parceiro íntimo e depressão em estudantes da Universidade Autônoma de Guerrero, no México, durante a pandemia COVID-19. A pesquisa é mista, questionários com respostas de múltipla escolha derivados do Inventário de Depressão de Beck e do Teste de Violência 
foram aplicados. A população era composta por 737 alunos, 564 eram mulheres e 173 homens. Destes, 71,91\% expressaram ter relacionamento saudável com o parceiro, 8,82\% apresentaram violência leve e 6,92\% violência grave, porém 29,44\% apresentaram ideação suicida. Os resultados indicam que os universitários apresentaram casos de depressão e apenas um quarto apresentou pensamentos suicidas. Isso significa que a crise de saúde afetou o estado mental dos alunos e com isso um problema acadêmico que deve ser resolvido pelas autoridades universitárias.

PALABRAS CLAVE: Depressão, estudantes, pandemia, violência.

\section{INTRODUCCIÓN}

La violencia de pareja es un fenómeno que se experimentan en todos los sectores sociales y culturales del mundo (Cáceres, 2004). Particularmente las mujeres son las que más sufren, aunque también se sabe que los hombres lo padecen. Sin embargo, el nivel de proporción experimentado por las mujeres es mucho mayor debido a la manera en que está organizada la sociedad (falocéntrica o machista) y al modo en que las instituciones invisibilizan este problema. Por ello, las instituciones han implementado políticas públicas en pro de su prevención y atención, pero, debido a las contradicciones sociales que existen y a los roles (complejos y verticales) que cumplen tanto varones como mujeres ha sido difícil su desarrollo. Expuesto esto, en el presente artículo se estudia la violencia de pareja y depresión que experimentan los estudiantes de la Universidad Autónoma de Guerrero (UAGro) en Acapulco, en el contexto de la COVID-191.

Cabe mencionar que en Guerrero entre el 2018 y 2020 la población en situación de pobreza fue mayor o igual al 51.1\% y la pobreza extrema fue de 16.2\% (Consejo nacional de evaluación de la política de desarrollo social, 2020). La situación durante la pandemia no ha sido de las mejores, todo parece indicar que se ensancharon más las desigualdades sociales y económicas. No obstante, a pesar de las enormes disparidades económicas y sociales los jóvenes de 18 y 40 años consideran que una de las formas para lograr incorporarse al mercado del trabajo es continuar sus estudios en la universidad.

En ese sentido, se ha notado que la pandemia ha invisibilizado un tema poco discutido y tiene que ver con las relaciones emocionales que se dan entre los jóvenes universitarios, nos referimos a la violencia de pareja y la depresión. Esta etapa emergente o adultez

\footnotetext{
1 Queremos agradecer por este trabajo a la Mtra. Aleyda Alejandra Hernández Ojeda (antares-
} 79@hotmail.com) y a la Lic. Grecia Lilian Alejandra Román Quevedo (cebollita_titi08@hotmail.com). 
temprana que caracteriza a dichos estudiantes como la apertura de posibilidades diversas representa un periodo de fuertes responsabilidades, ya que precisamente en este lapso es cuando la persona forma una pareja y familia, además consolida su profesión y trabajo para enfrentar al futuro con mejores herramientas. A pesar de que el proceso puede ser muy angustiante, los jóvenes pueden llenarse de incertidumbre y confusión, sin embargo, la mayoría de ellos tienen una visión positiva de su futuro e intentan a superar sus dificultades (Uriarte, 2005).

De acuerdo con lo dicho, la edad adulta temprana abarca del periodo de 20 a 40 años, incluso la considera como la etapa de plenitud del individuo. Los retos que enfrentan los estudiantes en este periodo consisten en concluir su profesión para poder enfrentar el futuro con mejores oportunidades (Uriarte, 2005). En este periodo el joven se preocupa por mantener una relación de pareja para poder consolidar y formar una familia. Dicho de otra manera, los avances de la sociedad moderna tienen que ver con tres tipos de confianza, que están en relación hacia uno mismo, otras personas y las instituciones. De acuerdo con esto, los males de la era moderna tienen que ver con la falta de confianza en una o en los tres tipos de seguridad que tiene el individuo. Por eso, los jóvenes carecen de una seguridad hacia sí mismos y esto hace que no logren relacionarse con las demás personas de manera permanente. Hoy en día hay cada vez más jóvenes prefieren adoptar una mascota que comprometerse con tener un hijo. Algo similar sucede con su relación con las instituciones, puesto que muchos de estos no logran permanecer en un trabajo fijo. En ese sentido, los jóvenes cada vez se comprometen menos, esto implica hablar de una era líquida, la cual está caracterizada por una forma de vida social marcada por la incertidumbre y el miedo al futuro, resultado de la globalización (Vásquez, 2008).

En relación a lo expuesto, la persona que por iniciativa propia ingresa a los estudios universitarios partiendo de su vocación, acude a la universidad para formarse profesionalmente (González, 2003). Ciertamente, en esta etapa juvenil ya cuentan con alguna pareja o la adquieren en la universidad. Se ha visto que en esta institución se llevan a cabo relaciones complejas entres las parejas a tal grado de que existe un uso intencional de la fuerza. La Organización Mundial de la Salud señala que la violencia en este tipo de circunstancias puede entenderse como el uso intencional de la fuerza física o el poder, ya sea en grado de coacción contra uno mismo, otra persona o una comunidad que tenga 
muchas probabilidades de causar lesiones, muerte, daños psicológicos, trastornos del desarrollo o privaciones(OMS, 2002).

La violencia de pareja puede ser provocada por conductas suicidas o por un grupo de personas que por lo general ocasionan daños psicológicos o problemas en el desarrollo, sobre todo, de las mujeres. La violencia contra ellas, en especial la que es ejercida por su pareja, como la violencia sexual, son un grave problema de salud pública y social en el mundo, que viola los derechos humanos de las mujeres. Una de cada tres mujeres en el mundo ha sufrido violencia de pareja, de tipo física o sexual, o padecido de violencia sexual por terceras personas en algún momento de su vida (Krug et al., 2003; OMS, 2002). Por lo anterior, a nivel mundial, el 27\% de las mujeres entre 15 y 49 años que han vivido en relación de pareja expresan haber sufrido algún tipo de violencia física y/o sexual (Krug et al., 2003). Por otro lado, la depresión es un trastorno mental frecuente, se estima que afecta a más de 300 millones de personas en todo el mundo y menciona que se puede convertir en un problema serio de salud pública, sobre todo cuando es de intensidad moderada a grave, porque puede alterar las actividades familiares, escolares y laborales, incluso, puede llevar al suicidio. Precisamente, en la presente investigación se estudiarán los participantes que resulten con síntomas de depresión, mismos que arrojará el Inventario de depresión de Beck Il (Sanz et al., 2003).

El 31 de diciembre de 2019 la OMS recibió diversos comunicados y fue notificado de manera concreta de la existencia de varios casos de neumonía viral atípica producida en Wuhan. Luego de esto, con el correr de los meses, diversos países fueron afectados por una enfermedad viral que hizo que, en febrero del 2020, el director general de esta organización lo declara como una pandemia mundial (Peña \& Rincón, 2020). Se supo que se trataba de una enfermedad infecciosa que pertenecía a la familia del coronavirus, sus síntomas más frecuentes eran fiebre, tos seca y cansancio. Esta enfermedad generó una crisis sanitaria mundial y afectó muchas instituciones estatales. Una de esas era la universidad, donde tanto docentes como estudiantes entraban a una fase de histeria, depresión e incomprensión por el fenómeno que se venía dando. Muchos de ellos empezaron a sufrir estrés crónico debido a que sus vidas cotidianas se habían visto reducidas a ciertas políticas de control, así como a diversas actividades teledirigidas o trabajos académicos en línea. En el caso de Guerrero, 
el gobernador había declarado el cierre de los espacios públicos y el rector de la universidad el trabajo desde casa. Dicho esto, en este artículo se estudia el aumento de la violencia de pareja y se mide la depresión durante la pandemia.

\section{MÉTODO}

La investigación es mixta con cuantificación y cualificación de datos, además es de corte transversal, porque el estudio se realizó durante el inicio de la pandemia del coronavirus (Sanz et al., 2003). La población se constituyó por 737 estudiantes, de los cuales 564 (76.52\%) son mujeres, mientras que 173 (23.48\%) corresponde a hombres. Cabe indicar que el criterio a trabajar fue con base en un muestreo voluntario y la característica esencial tomada en cuenta fue considerar que todos los participantes sean estudiantes de la Universidad Autónoma de Guerrero, México.

Los datos fueron cargados y analizados en hoja de cálculo de Excel. El instrumento utilizado fue el Cuestionario de Violencia, se trata de una prueba no estandarizada y aún no validada, sin embargo brinda herramientas importantes para que una persona que se halle viviendo una relación de violencia la pueda identificar por sí misma (Consejo nacional de igualdad de género, 2014). Cabe añadir que también se utilizó el Inventario de Depresión de Beck II, el cual consiste en un test autoaplicable que consta de 21 ítems y evalúa la gravedad de síntomas depresivos en adolescentes y adultos (Beck, 2014; Beltrán et al., 2012; Sanz et al., 2003). Muestra una fiabilidad y consistencia interna excelente, además de un cuestionario con respuestas de opción múltiple de propia creación que recoge datos personales y que muestra actividades cotidianas que realizan con estereotipos de género mismas que respondieron los estudiantes de forma virtual.

El Test de violencia es un cuestionario que fue realizado por el Consejo Nacional de Igualdad de Género (2014) el cual consta de tres secciones y un total de 33 preguntas. La primera parte identifica las primeras fases de violencia. La segunda visibiliza la necesidad de ayuda o auxilio en relaciones violentas más graves y la tercera pone en evidencia el peligro que pudiera llegar a correr quien está contestando el cuestionario. Es una herramienta que permite a las y los estudiantes en situación de violencia por parte de su pareja a identificar 
por sí mismos si viven violencia y en qué grado. En términos generales se podría decir que se trata de un test estandarizado sencillo en su aplicación y respuesta. El Cuestionario de Violencia se integra de tres fases de las cuales, para los fines del estudio, se aplicaron la Fase 1, Fase 2, y Fase 3, constan de 10, 11 y 12 ítems respectivamente. Cada ítem presenta tres posibilidades de respuesta que se contestan con base en la siguiente escala: se asigna 1 para "Nunca", 2 para "En Ocasiones", y 3, para "Casi Siempre". El puntaje máximo que se puede registrar en la Fase 1 es 30, en la Fase 2 es de 33, mientras que en la Fase 3 es de 36 puntos. La Fase 1 representa violencia en fase mínima, la Fase 2 moderada y la Fase 3 violencia grave.

El Inventario de Depresión de Beck es un instrumento utilizado en el campo clínico que sirve para evaluar indicadores de depresión, en cuanto a la confiabilidad presenta un coeficiente de alfa de 0,88 y el inventario demuestra validez interna y externa (Beck, 2014). Consta de 21 ítems que mide indicadores de depresión en adolescentes mayores de 13 años y adultos. Cada ítem se evalúa en una escala de cuatro puntos que va desde el cero hasta el tres, al final se suman dando un total que puede estar entre cero y 63. El Inventario de Depresión (BDI-II) es útil para diagnosticar cuatro niveles del padecimiento clínico, desde la depresión mínima (de 0 a 13), depresión leve (de 14 a 19), depresión moderada (de 20 a 28) y la depresión severa (de 29 a 63).

En cuanto a los procedimientos de aplicación se procedió de la siguiente manera: la administración de los instrumentos se hizo a distancia, se envió a los estudiantes de psicología el enlace para que abrieran los archivos, leyeran las instrucciones, contestaran y regresaran la emisión de sus respuestas a la oficina emisora/receptora.

\section{RESULTADOS}

El Test de violencia y el Inventario de Depresión sirvieron para comprender la relación entre la violencia de pareja y la depresión en la población estudiantil de la Universidad Autónoma de Guerrero. Para realizar este estudio fue necesario aplicar encuestas con respuestas de opción múltiple. Debemos señalar que este trabajo se efectuó durante la pandemia. La población estaba constituida por 737 estudiantes, de los cuales 564 eran mujeres y 173 
hombres. A continuación, se muestran los resultados que derivaron de ambos instrumentos usados en estudios de psicología clínica.

\section{Tabla 1}

Resultados de violencia de pareja y depresión estudiantil

PASE AMARILLA

1. Te culpa por sus cambios de ánimo Porcentaje

2. ¿Te amenaza o chantajea?

$11.4 \%$

3. ¿Te grita, se burla de ti, te humilla?

$5.7 \%$

4. Desestima tus opiniones y te critica

$5.1 \%$

5. Controla tu forma de vestir

$9.9 \%$

6. ¿Te prohíbe decidir sobre tu dinero y tus bienes?

$9.4 \%$

7. Controla tu hora de salida, de llegada

$6.8 \%$

$15.3 \%$

9. ¿Tu relación de pareja te ha llevado a ser destructiva?

$8.3 \%$

10. ¿Tu pareja se irrita si le dices que fuma? o bebe demasiado? $\quad 7.8 \%$

FASE NARANJA

$4.9 \%$

1. ¿Te empuja, te golpea, te pellizca?

$2 ¿$ Evitas tocar ciertos temas o hacer ciertas cosas por temor a

$8.3 \%$

las reacciones de tu pareja?

3. ¿Sientes culpa o responsabilidad por las reacciones violentas

$5.7 \%$ de quien te agrede?

4. ¿Tienes conocimiento si tu pareja ha violentado a parejas

$5.6 \%$ anteriores?

5. ¿Tu pareja es excesivamente celosa y posesiva?

$10.4 \%$

6. ¿Te has aislado de tu familia, amigas y amigos?

$9.9 \%$

7. ¿Crees que con tu sacrificio tu pareja pueda cambiar?

$18.3 \%$

8. ¿Sientes que tu pareja tiene la razón en sus críticas, quejas o

$10.3 \%$ reclamos contra ti?

\section{FASE ROJA}

7. ¿Estás deprimida y con problema de salud, o has tenido

$11 \%$ pensamientos suicidas?

11. ¿Sientes que nadie te puede ayudar o no consideras que

$6.4 \%$ mereces ayuda?

Nota: Elaboración propia

Entre los hallazgos encontrados en el Test de Violencia Fase I, sobre todo, en la variable que está relacionado con culpar a alguien por su estado de ánimo, el 14.11\% contestó que esto ocurre en ocasiones y casi siempre; en cuanto al control de la hora de salida o de llegada a la universidad, el 22.25\% respondió que en ocasiones y casi siempre existe una crisis emocional. En la Fase 2 del mismo test, al abordar los celos, el 10.04\% respondió que su pareja es celosa, en ocasiones. Respecto al sacrificio que hace la pareja para cambiar su 
forma de ser y actuar, el 17.78\% respondió que solo en ocasiones. Por su parte, el Inventario de Depresión (BDI II), demostró que el 11.13\% de los estudiantes resultó con depresión leve, el $8.68 \%$ con depresión moderada y el $9.77 \%$ con depresión severa; la suma de porcentajes parciales indica que el $29.58 \%$ de los participantes padece depresión en sus diferentes niveles. A manera de conclusión se declara que la violencia de pareja, sí influye al aumentar los niveles de depresión, en el contexto del COVID-19 (OMS, 2002; Peña \& Rincón, 2020).

Los instrumentos que contestaron los estudiantes reportaron los siguientes resultados: las respuestas del test de violencia en la fase 1: al indicador de culpar a alguien por los estados de ánimo, el $14.11 \%$ contestaron que en ocasiones y casi siempre. En ese tenor, en cuanto al control de la hora de salida o de llegada, el 22.25\% respondieron que en ocasiones y casi siempre. Con relación a la Fase 2 del mismo test, al abordar los celos el 10.04\% respondió que en ocasiones. Respecto al sacrificio que hace la pareja para cambiar su forma de ser y actuar, el $17.78 \%$ respondió que en ocasiones. Por lo tanto, el $8.82 \%$ de los examinados mostró sufrir violencia en la Fase 1 (leve), el 12.35\% violencia en la Fase 2 (moderada), mientras que el 6.92\% manifestó experimentar violencia en la Fase 3 (grave).

Fig. 1

Resultados de los niveles de depresión

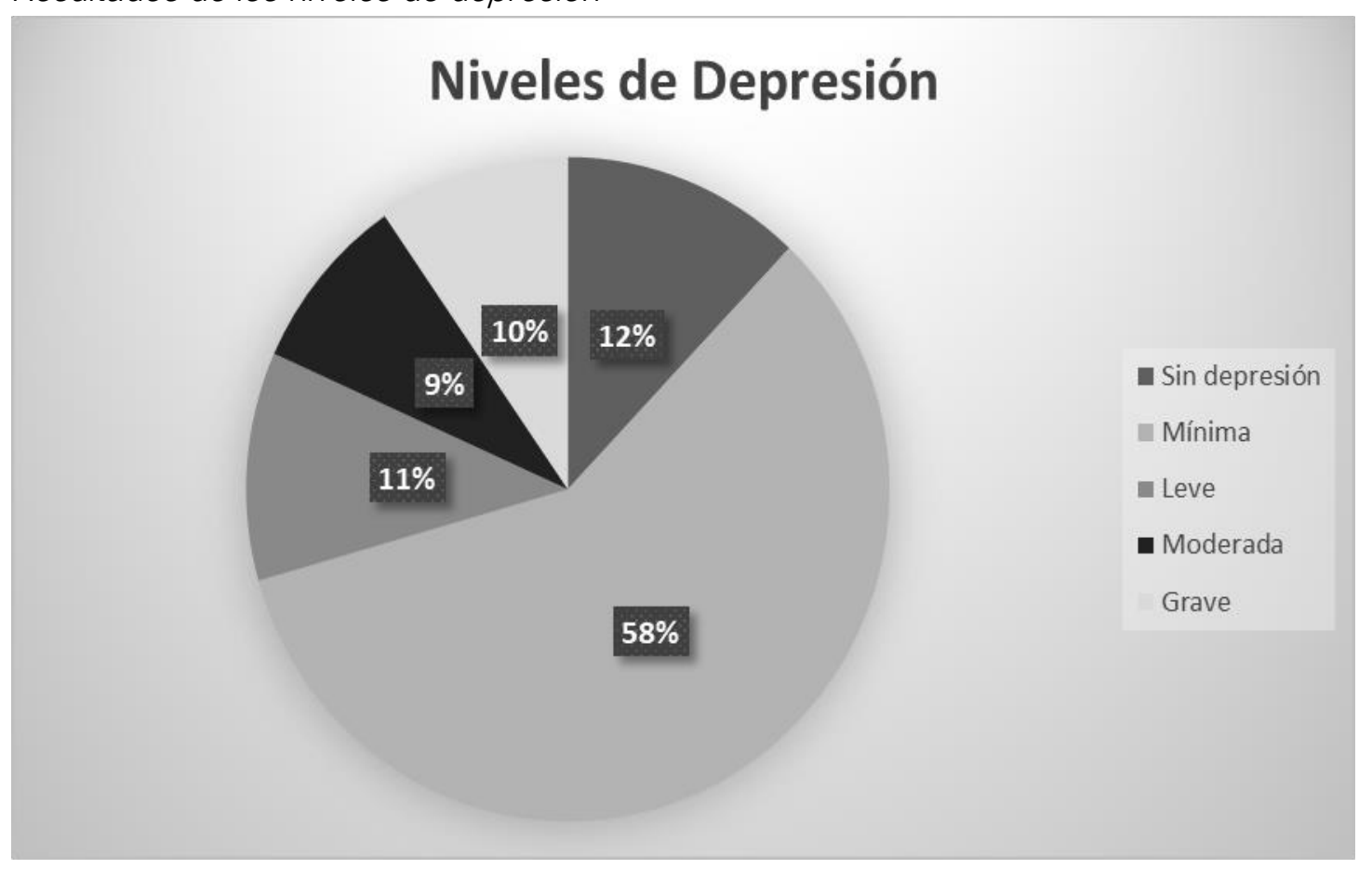

Nota: elaboración propia 
En ese sentido, el 3.53\%, de estudiantes mostraron sufrir violencia en las tres fases. Por otra parte, en el inventario de depresión (BDI II), resultaron el 12.08\% sin indicadores de depresión, mientras que, el 58.48\% de los examinados resultó con depresión mínima. Por otra parte, el $11.13 \%$ se ubica en depresión leve; asimismo, el $8.68 \%$ de estudiantes presentaron depresión moderada y el 9.63\% mostró depresión grave; haciendo un total de 87.92\% de participantes resultantes con algún tipo de depresión (ver figura 2). Asimismo, el $21.98 \%$ de los participantes, mostraron pensamiento suicida. Además, 13 estudiantes, el 1.76\% presentaron síntomas de violencia en las tres fases aunado a depresión grave; cuatro más, el 54\% reflejaron violencia en las tres fases con depresión moderada y tres más, el $41 \%$ mostraron violencia en las tres fases y depresión leve. Sin embargo, 14 estudiantes, el $1.90 \%$ presentaron depresión grave y alguna fase de violencia de pareja, 16 participantes del estudio, el $2.17 \%$ mostraron violencia en alguna fase y depresión moderada y 17 estudiantes, el $2.31 \%$ resultaron con violencia en alguna fase y depresión leve (Acuña et. al., 2020).

\section{DISCUSIÓN}

Se declara que la violencia de pareja, sí influye al aumentar los niveles de depresión. En el estudio de violencia en parejas de jóvenes universitarios durante la pandemia por COVID19, se encontró que predominan los celos, así como la vigilancia hacia la pareja, esto aunado a una correlación entre celos, control sufrido que es cometido por las personas que exigen y/o construyen una relación amorosa (Beck, 2014; Beltrán et al., 2012; Sanz et al., 2003). Este fenómeno no es para menos, puesto que la pandemia como un problema inherente a la crisis civilizatoria ha generado una total reconfiguración no sólo los entornos materiales, sino también psicológicos (Acuña et. al., 2020). Los efectos de esta crisis sanitaria se manifiestan a partir de rupturas sociales y, sobre todo, mentales que impiden el desarrollo pleno del ser humano. Se trata de enfermedades como la depresión o el suicidio que alteran no sólo el estado anímico de las personas, sino también que afectan al colectivo.

Este hecho es alarmante y debe ser motivo de preocupación, porque, si los jóvenes tienen depresión o intentan inmolarse lo que va a ocurrir es una crisis individual y colectiva, pero no sólo eso, esto puede convertirse en un fenómeno anómico (Lopez, 2009). Nos parece importante indicar que estos casos donde los jóvenes se quitan la vida y revelan su malestar 
a través de actos e ideaciones suicidas, si bien no discutidas y reconocidas como actos de catarsis o crisis socioemocional, manifiestan las ausencias de políticas de salud mental que vayan en un sentido restaurador y no meramente clínico. Dicho esto, creemos que el estado mental, sobre todo, en las instituciones de educación superior, es fundamental para que el tejido social no se desestructure. La problemática que se ha visto reflejada en las aulas de la Universidad Autónoma de Guerrero es sólo una muestra de las enfermedades postpandemia que deben de ser atendidas, porque una de las mayores responsabilidades de las instituciones es velar no sólo de la reproducción de los sistemas políticos, sino también el de poner en el centro del debate al sujeto. Esto implica considerar no sólo la existencia de éste como un ser cosificado o atrapado por los ciclos de la violencia cotidiana, sino también como un ser con potente y frágil ante situaciones en las cuales nos encontramos.

Es necesario seguir discutiendo el tema de la violencia (en plural) generada por la pandemia, así como sus efectos lamentables en el individuo. Específicamente, nos referimos a la depresión, porque se trata de un trastorno o enfermedad mental no visible por las autoridades. Negar su existencia implicaría convertir este problema en un verdadero fenómeno social que afectaría a la sociedad y la economía; asimismo, el Estado se vería en la obligación de invertir más en infraestructura o, en su defecto, poner en marcha políticas de salud que atiendan estos problemas emocionales que impiden el buen funcionamiento orgánico de la sociedad. El trabajo expuesto es un aporte para prevenir una posible masificación de casos, pero también un puente para discutir temas tan actuales que ha sido provocado por la crisis sanitaria.

\section{CONCLUSIÓN}

Para terminar, 530 estudiantes, el $71.91 \%$ de la UAGro Acapulco, mostraron en general que tienen relaciones saludables de pareja en el contexto de COVID-19, sólo 51 estudiantes mostraron que experimentaron violencia moderada, 65 , el $8.82 \%$ de los examinados presentó violencia leve y 51 estudiantes, el 6.92\% experimentaron violencia grave. En cuanto a participantes que presentaron síntomas de depresión, fueron 217, el 29.44\%, de los cuales 16 mostraron ideación suicida.

En cuanto a los resultados del test de depresión durante la pandemia casi la tercera parte 
de la población estudiantil mostró indicadores de depresión en alguno de sus tipos, además, aproximadamente la cuarta parte de los examinados mostró pensamiento suicida. En ese sentido, se puede afirmar que no solo la violencia de pareja vivida por los estudiantes tiene relación con los síntomas de depresión, sino que en general los participantes mantienen sanas relaciones de pareja y convivencia. Sin embargo, el COVID-19, alteró la cotidianidad de la población estudiantil de la UAGro, misma que repercutió en su salud mental al resultar la mayoría de participantes, el 87.92\% con síntomas de depresión.

Es importante que se continúe evaluando y monitoreando los fenómenos psicológicos que se han mencionado, porque podría derivar en todo un problema de salud mental o, incluso, un problema de Estado. La crisis sanitaria ha trastocado la vida cotidiana, política y educativa y con esto acelerado de manera radical nuestras actividades diarias a un ritmo mayor. Esto implica considerar que la presión que hoy en día padecen los jóvenes y/o estudiantes del nivel superior se ha expresado en momentos de alteridad, histeria y/o paranoia colectiva. El solo hecho de pensar en suicidarse o quitarse la vida es una de esas. Actualmente se manifiestan nuevos malestares y trastornos individuales y sociales, pero nuevas líneas de trabajo e investigación que expliquen a ciencia cierta cuáles son los orígenes de todos estos síntomas. Con esta investigación, esperamos abrir la discusión en torno a la violencia (moderna) y sus efectos en los cuerpos y mentalidades.

\section{REFERENCIAS}

Acuña, K, Sánchez, E., y Zepeda, Manuel (2020). Cartografías de la pandemia en tiempos de crisis civilizatoria. Aproximaciones a su entendimiento desde México y América Latina (La Bibliot).

Beck, A. (2014). ( PDF) Inventario de depresión de Beck. PAIDOS.

Beltrán, M. del C., Freyre, M. Á., \& Hernández-Guzmán, L. (2012). The beck Depression Inventory: Its validity in adolescent population. Terapia Psicologica, 30(1), 5-13. https://doi.org/10.4067/s0718-48082012000100001

Cáceres, J. (2004). Violencia física, psicológica y sexual en el ámbito de la pareja : papel del contexto Physical, psychological and sexual violence within the couple: the role of the. Clínica y Salud, 15, 33-54.

CONEVAL. (2020). Informe de pobreza y evaluación 2020 Guerrero. 119. 
https://www.coneval.org.mx/coordinacion/entidades/Documents/Informes_de_pobreza _y_evaluacion_2020_Documentos/Informe_Guerrero_2020.pdf

Consejo Nacional de lgualdad de Género (2014). Test de violencia. https://repositorio.iaen.edu.ec/bitstream/24000/4471/1/TEST DE VIOLENCIA.pdf

González, N. et. al. (2003). Autoestima en jóvenes universitarios. Ciencias Humanas y de La Conducta, 173-179. http://ri.uaemex.mx/bitstream/handle/20.500.11799/38013/Autoestima en jóvenes universitarios. pdf?sequence $=1$ \&isAllowed $=y$

Krug, E. G., Dahlberg, L. L., Mercy, J. A., Zwi, A. B., \& Lozano, R. (2003). Informe mundial sobre la violencia y la salud. Revista Do Instituto de Medicina Tropical de São Paulo, 45(3), 130-130. https://doi.org/10.1590/s0036-46652003000300014

Lopez, M. del P. (2009). El Concepto de anomia de Durkheim y las aportaciones teóricas posteriores. Iberofórum. Revista de Ciencias Sociales de La Universidad Iberoamericana, M(8), 130-147.

Organizacion Mundial de la salud. (2002). Informe Mundial Sobre La Violencia Y La Salud. Organizacion Mundial de La Salud., 2-3, 1-11.

Peña-López, B. O., \& Rincón-Orozco, B. (2020). Generalidades de la Pandemia por COVID19 y su asociación genética con el virus del SARS. Revista de La Universidad Industrial de Santander. Salud, 52(2), 83-86. https://doi.org/10.18273/revsal.v52n2-2020001

Sanz, J., Perdigón, A. L., \& Vázquez, C. (2003). Adaptación española del Inventario para la Depresión de Beck-II ( BDI-II ): 2. Propiedades psicométricas en población general The spanish adaptation of Beck's Depression Inventory-II ( BDI-II ): Clinica y Salud, 14(3), 249-280.

Uriarte, J. de D. (2005). En la transición a la edad adulta. los adultos emergentes. International Journal of Developmental and Educational Psychology, 3, 145-160. https://www.redalyc.org/pdf/3498/349832310013.pdf

Vásquez, A. (2008). Modernidad Liquiday fragilidad Humana. Critical Journal of Social and Juridical Sciences, Juliodiciembre, 2008, 19 num 3, Publicación Electrónica de la Universidad Complute. http://www.redalyc.org/pdf/181/18101917.pdf 